\title{
Caroli disease and the central dot sign
}

\author{
Neil U. Lall • Mark J. Hogan
}

Received: 18 September 2008 /Revised: 4 December 2008 / Accepted: 7 January 2009 /Published online: 17 February 2009

(C) Springer-Verlag 2009

A 12-month-old boy presented with fever, Klebsiella bacteremia, emesis, and abdominal pain. US demonstrated multiple intrahepatic cysts containing fibrovascular bundles, known as the central dot sign (Fig. 1). Subsequent percutaneous transhepatic cholangiography showed segmental saccular biliary ductal dilation (Fig. 2).

Caroli disease is a rare congenital cystic dilatation of the intrahepatic bile ducts. This predisposes to biliary stasis and consequent lithiasis, cholangitis, abscesses, and septicemia. Additionally, children face increased risk of hepatic fibrosis (termed Caroli syndrome), renal disease, cholangiocarcinoma, and amyloidosis [2]. The central dot sign on US and CT is

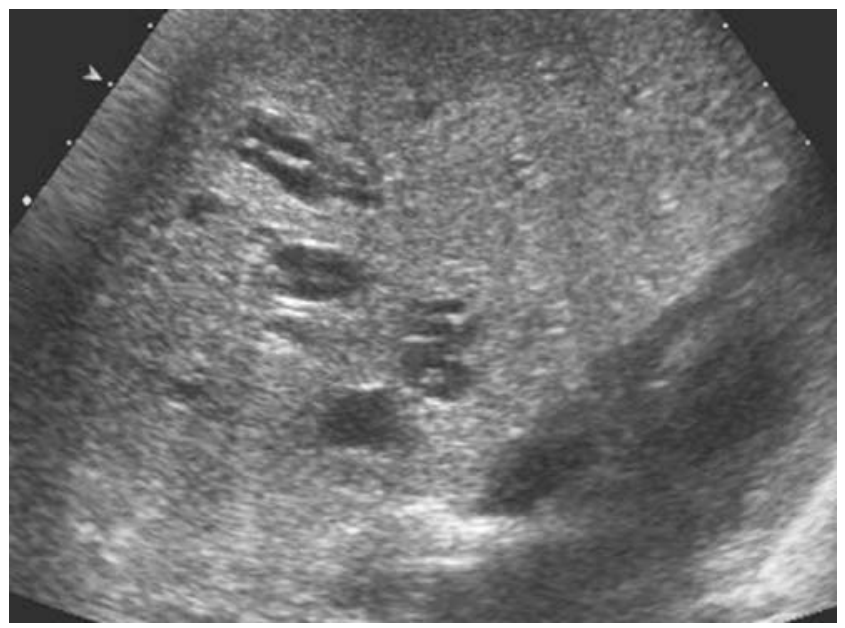

Fig. 1 Transverse US

\footnotetext{
N. U. Lall $(\bowtie)$

Department of Radiology, The Ohio State University,

150 Means Hall, 1654 Upham Drive,

Columbus, OH 43210, USA

e-mail: Lall.5@osu.edu

\section{J. Hogan}

Department of Radiology, Nationwide Children's Hospital,

Columbus, OH, USA
}

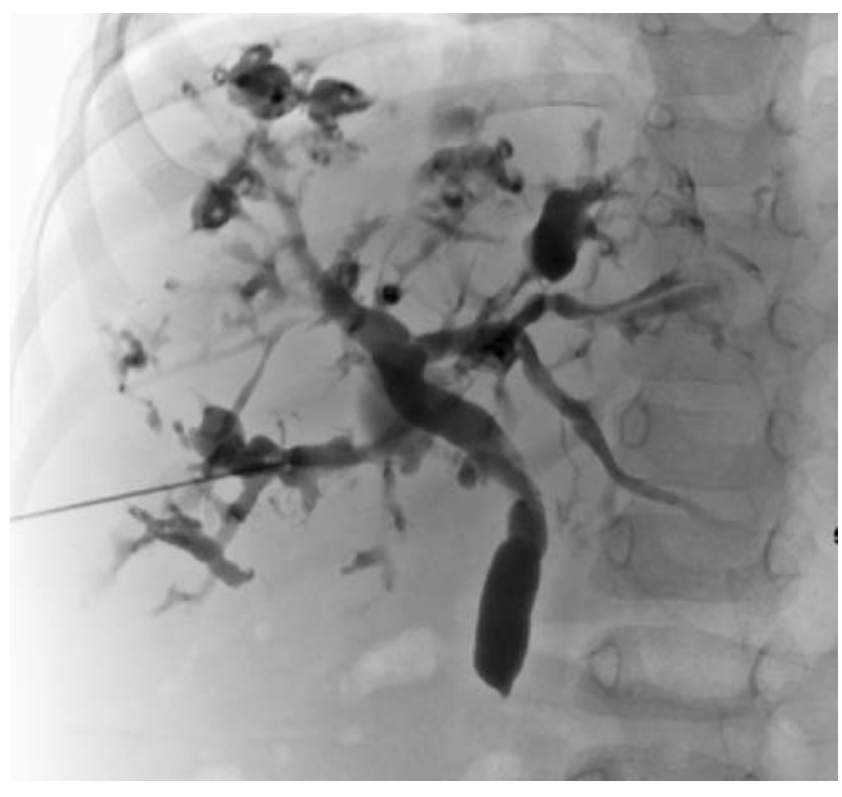

Fig. 2 Percutaneous transhepatic cholangiographic image

indicative of Caroli disease, but can also be seen in peribiliary cysts, obstructive jaundice, and periportal lymphedema [1]. Cholangiography is the most sensitive radiographic study, because communication between bile ducts and cystic loculations is important to differentiate from multiple liver cysts [2]; the role of magnetic resonance cholangiopancreatography for Caroli disease in children has not been systematically studied. Treatment can be achieved by partial hepatectomy in localized disease; however, diffuse liver involvement necessitates eventual transplant.

\section{References}

1. Ahmadi T, Itai Y, Minami M (1997) Central dot sign in entities other than Caroli disease. Radiat Med 15:381-384

2. Miller WJ, Sechtin AG, Campbell WL et al (1995) Imaging findings in Caroli's disease. AJR 165:333-337 